\title{
High dose immunoglobulin IV treatment in adrenoleukodystrophy
}

\author{
M Cappa, E Bertini, P del Balzo, P Cambiaso, A Di Biase, S Salvati
}

\begin{abstract}
$\mathrm{X}$-linked adrenoleukodystrophy (ALD) is an inborn error of peroxisomal metabolism characterised by progressive demyelination of the central nervous system and by hypoadrenalism. The biochemical defect of ALD results in an impairment in degradation of very long chain fatty acids (VLCFA) with their accumulation in plasma and tissues. Many therapeutic approaches have been tried. Recently, a restricted diet and glycerol trioleate/erucic (GTOE) supplementation have shown normalisation of VLCFA plasma levels, although they are not effective in altering the clinical course of X-linked ALD.
\end{abstract}

The preliminary results are presented of a twelve month trial of immunomodulation by intravenous high-dose immunoglobulins in six patients, mean (SD) age $10 \cdot 48(2 \cdot 8)$ affected by X-linked ALD, on VLCFA restricted diet plus GTOE supplementation therapy. Six patients aged $9.30(1.5)$ with similar clinical characteristics and on the same restricted VLCFA regime of GTO/GTE therapy were studied as the control group.

After two months VLCFA levels fell to normal values and remained so for all patients throughout the study. These data show that immunoglobulins are not able to arrest the progression of the disease. The MRI and clinical symptoms deteriorated to the same extent in both groups.

Reparto di

Endocrinologia e

Neurologia, $\star$ Ospedale

Bambino Gesù,

IRCCS, Rome, Italy

M Cappa

E Bertini*

P del Balzo

P Cambiaso

Instituto Superiore di

Sanitá, Rome, Italy

A Di Biase

S Salvati

Correspondence to:

DrE Bertini.
The leukodystrophies are a group of degenerative diseases involving the white matter. Enzymatic defects in myelin lipid metabolism lead to excessive tissue deposition of a normal component of myelin lipids or breakdown products of myelin. The clinical disorders include: metachromatic leukodystrophy, Canavan disease (spongy degeneration of the cerebral white matter), Krabbe's disease (globoid leukodystrophy). PelizaeusMerzbacher and adrenoleukodystrophy. Xlinked adrenoleukodystrophy (ALD) is an inherited disorder caused by a gene located in Xq28 and coding for an ATP-binding transporter. ${ }^{1}$ This disorder is characterised by an intraperoxisomal enzymatic defect leading to accumulation of very long chain fatty acids (VLCFA) in the central nervous system and adrenal glands. CNS demyelination and adrenal insufficiency are major features of this disorder. ${ }^{2-3}$ Adrenoleukodystrophy has a wide phenotypic variability, but most cases appear in childhood or adolescence as devastating degenerative disorders leading to major neurological deterioration and death within a few years.

Neurological symptoms often precede signs of adrenal insufficiency, but $70-80 \%$ of the neurologically involved cases show slight adrenal insufficiency revealed by impaired cortisol response to exogenous ACTH. ${ }^{2}$

Many therapeutic attempts have been tried including plasmapheresis, dietary restriction, bone marrow transplantation (BMT), dietary restriction plus glycerol trioleate acid (GTO) and dietary restriction plus a mixture of GTO and erucic acid (GTOE; Lorenzo's oil). ${ }^{2}$ None of them are completely effective and although some encouraging results have been obtained with BMT and GTOE, there is currently no effective treatment for ALD. ${ }^{4-6}$ Clinical improvement following intravenous gammaglobulin therapy has been reported in $\mathrm{ALD}^{7}$ and immunoglobulins may play a role in repairing myelin. ${ }^{8}$ For this reason we included six childhood and adolescent ALD patients in our trial with intravenous immunoglobulins (IVIg).

\section{Methods}

PATIENTS

Among our series of ALD patients (63 cases), at Bambino Gesú Children's Hospital, we observed 12 patients affected by X-linked childhood and adolescent ALD between January 1991 and June 1993. The diagnosis was confirmed by measuring VLCFA in plasma considering the absolute amount of C26:0 and $\mathrm{C} 26 / \mathrm{C} 22$ ratio, using a previously described method. ${ }^{4}$

The patients were randomly subdivided into two groups according to their age and grade of neurological status (Kurtzke score less than or equal to 5) ${ }^{9}$ : Group $1-\mathrm{Six}$ patients aged between 7.9 to 14 years were given VLCFA restricted diet plus GTOE oil supplementation therapy, plus intravenous gammaglobulin therapy. Group 2-Six patients aged between seven to 12.6 years were given VLCFA restricted diet plus GTOE oil supplementation therapy.

All patients were admitted to the Department of Endocrinology of the Bambino Gesú Hospital under an institutionally approved protocol for the study. Written informed consent was obtained from all subjects and/or their guardians. 
Mean (SD) results of the patients in the study. Patients 1-6 (group 1) on IG + diet therapy; patients 7-12 (group 2) diet alone

\begin{tabular}{|c|c|c|c|c|c|c|c|}
\hline \multirow[b]{2}{*}{ Patient } & \multirow{2}{*}{$\begin{array}{l}\text { Age } \\
\text { year/month }\end{array}$} & \multicolumn{2}{|c|}{ Kurtzke Score } & \multicolumn{2}{|l|}{$M R I$} & \multicolumn{2}{|c|}{$V L C F A$ ug/ml } \\
\hline & & base & after & base & after & base & after \\
\hline $\begin{array}{l}1 \\
2 \\
3 \\
4 \\
5 \\
6\end{array}$ & $\begin{array}{l}11 \\
11 \cdot 6 \\
7 \cdot 9 \\
8 \cdot 6 \\
14 \cdot 0 \\
9 \cdot 8\end{array}$ & $\begin{array}{l}1 \cdot 0 \\
3 \cdot 5 \\
2 \cdot 0 \\
1.5 \\
2 \cdot 5 \\
3 \cdot 5\end{array}$ & $\begin{array}{l}1 \cdot 5 \\
5 \cdot 5 \\
9 \cdot 5 \\
7 \cdot 5 \\
9 \cdot 0 \\
7 \cdot 0\end{array}$ & $\begin{array}{l}+ \\
+ \\
+ \\
+ \\
+ \\
+\end{array}$ & $\begin{array}{l}++ \\
++ \\
++ \\
+ \\
++ \\
+\end{array}$ & $\begin{array}{l}1 \cdot 4 \\
1 \cdot 2 \\
1 \cdot 1 \\
0.95 \\
1 \cdot 5 \\
1 \cdot 4\end{array}$ & $\begin{array}{l}0 \cdot 8 \\
0 \cdot 35 \\
0 \cdot 5 \\
0 \cdot 35 \\
0 \cdot 5 \\
0 \cdot 4\end{array}$ \\
\hline $\begin{array}{r}7 \\
8 \\
9 \\
10 \\
11 \\
12\end{array}$ & $\begin{array}{l}10 \cdot 4(2 \cdot 2) \\
11 \\
8 \\
10 \\
7 \\
12 \cdot 6 \\
7 \cdot 2\end{array}$ & $\begin{array}{l}2 \cdot 3(1 \cdot 0) \\
1 \cdot 0 \\
2 \cdot 5 \\
5 \cdot 0 \\
5 \cdot 5 \\
2 \cdot 5 \\
4 \cdot 5\end{array}$ & $\begin{array}{l}6 \cdot 7(2 \cdot 9) \\
1 \cdot 0 \\
9 \cdot 0 \\
9 \cdot 0 \\
9 \cdot 0 \\
2 \cdot 5 \\
5 \cdot 5\end{array}$ & $\begin{array}{l}+ \\
+ \\
+ \\
+ \\
+ \\
+\end{array}$ & $\begin{array}{l}+ \\
++ \\
++ \\
++ \\
+ \\
++\end{array}$ & $\begin{array}{l}1 \cdot 26(0 \cdot 21) \\
2 \cdot 1 \\
1 \cdot 6 \\
1.4 \\
1 \cdot 2 \\
1.5 \\
0.9\end{array}$ & $\begin{array}{l}0.48(0 \cdot 17) \\
0.4 \\
0 \cdot 5 \\
0 \cdot 4 \\
0 \cdot 6 \\
0 \cdot 5 \\
0 \cdot 3\end{array}$ \\
\hline & $9 \cdot 3(2 \cdot 27)$ & $3.3(1.57)$ & $6.0(3.6)$ & & & $1.45(0.4)$ & $0.45(0 \cdot 1)$ \\
\hline
\end{tabular}

EDSS score (Kurtzke score): 3.0 mild disability; 3.5 moderate disability; 4.0 self-sufficient for up to 12 hours; 4.5 require minimal assistance, has some limits on activity but able to work a full day; 5.0 severe disability, cannot perform full day's activity, but ambulatory without aid; 5.5 severe disability precluding full day's activities, walks about 100 metres; 6 requires unilateral assistance to walk 100 metres; 6.5 requires bilateral assistance for walking 20 metres; 7 walks less than 5 metres and essentially restricted to a wheelchair; 7.5 restricted to a wheelchair; 8 restricted to bed or chair; 9 vegetative state.

Change of 0.5 was considered stable condition.

CLINICAL EVALUATION

The patients had a standard physical examination, electrocardiography, echocardiography, biochemistry comprising electrolytes, liver-enzyme, platelet leukocyte counts on admission and every three months. VLCFA and $\mathrm{C}: 26 / \mathrm{C}: 22$ ratios were assessed at admission and every three months. The Kurtzke expanded disability status scale (EDSS) was recorded at diagnosis and at six and 12 months. MRI and BAERs were performed at diagnosis and after 12 months.

\section{STUDY PROTOCOL}

All patients followed a restricted VLCFA $\operatorname{diet}^{10}$ with GTOE supplementation (40 mg/ day). Six patients received high dose intravenous ( $1 \mathrm{~g} / \mathrm{kg}$ bw in $8-12$ hours) gammaglobulin (Merieux-France) infusions every 15 days for three months and every month for a year, thereafter.

\section{Results}

All patients showed a normalisation of VLCFA within three months and in all but one (patient 1), we observed normal levels of VLCFA throughout the study. Patient number 1 withdrew from the diet at nine months. The table summarises the results obtained. In both groups there was a deterioration of neurological symptoms as revealed by the EDSS score. Only two patients ( 7 and 11) showed no clinical change (less than 0.5 of EDSS). An increase of detected T2 signal abnormality was observed in eight of 12 patients (four for each group). Auditory conduction in the brainstem (wave $I-V$ interpeak latency) was delayed in all patients, and it remained unchanged in both groups of patients.

Thrombocytopenia was observed in $50 \%$ of patients and transient elevation of transaminases was observed in both groups (side effects were due to GTOE administration). Fever after IVIg (75\% of cases) and seizures with fever after IVIg (in 1 case) were also observed.

\section{Discussion}

Despite plasma normalisation of VLCFA, the neurological symptoms of this disorder deteriorated in all the reports that have so far been published. ${ }^{4}$ The reversal of early neurological and neuroradiological manifestations in adrenoleukodystrophy following bone marrow transplantation is not explained by a decrease of serum VLCFA because these levels can remain high after BMT (reference 5 and personal experience).

Factors other than metabolic abnormalities appear to be involved in the myelin damage in this disorder. Degenerative changes in fibre tracts that affect both axons and myelin sheaths are accompanied by striking perivascular accumulations of inflammatory cells, ${ }^{3}$ supporting the hypothesis that immunological factors are involved in the pathogenesis of this disease.

Our preliminary results are not encouraging and currently we do not know why immunomodulation does not determine clinical improvement in our patients.

1 Mosser J, Douar AM, Sarde CO, et al. Putative X-linked adrenoleukodystrophy gene share unexpected homology with ABC transporters. Nature 1993;361:726-30.

2 Moser HW, Moser AB. Adrenoleukodystrophy (Xlinked). In: Scriver CR, Beadet AL, Sly WS, Valle D, eds. The metabolic basis of inherited disease 6th ed, vol 2 . New York: McGraw-Hill, 1989;1511-32.

3 Schaumburg HH, Powers JM, Raine CS, et al. Adrenolekodystrophy. Arch Neurol 1975;32:577-91.

4 Uziel G, Bertini E, Bardelli, et al. Experience on therapy of adrenoleukodystrophy and adrenomyeloneuropathy. Dev Neurosci 1991;13:274-9.

5 Aubourg P, Blanche S, Jambaque I, et al. Reversal of early neurological manifestations of X-linked adrenoleukodystrophy by bone marrow transplantation. $N$ Engl f Med 1990;322:1860-65.

6 Aubourg P, Adamsbaum C, Lavallard-Rousseau MC, et al. A two year trial of oleic and erucic acids (Lorenzo's oil) as treatment for adrenomyeloneuropathy. $N$ Engl $\mathcal{f}$ Med 1993;329:745-52.

7 Miike T, Taku K, Tamura T, et al. Clinical improvement of adrenoleukodystrophy following intravenous gammaglobulin therapy. Brain Dev 1989;11:134-7.

8 Rodriguez M, Lennon WA. Immunoglobulins promote remyelination in the central nervous system. Ann Neurol remyelination in

9 Kurtzke JF. Rating neurological impairment in multiple sclerosis: an expanded disability status scale (EDDS). sclerosis: an expanded disab

10 Gambarara M, Del Balzo P, Cambiaso $P$, et al. Therapeutical approaches of ALD: problems with diet in Italy. In: Uziel G, Wanders RJA and Cappa M, eds. Adrenoleukodystropy and other peroxisomal disorders. Amsterdam: Elsevier (Biomedical Division), 1990:181-93. 